\title{
Steady flow of an incompressible perfectly conducting fluid past a thin airfoil
}

Adrian Carabineanu*

\author{
*Correspondence: \\ acara@fmi.unibuc.ro \\ Department of Mathematics, \\ University of Bucharest, Academiei \\ 14, Bucharest, Romania \\ Institute of Mathematical Statistics \\ and Applied Mathematics, \\ Romanian Academy, Calea 13 \\ Septembrie 13, Bucharest, Romania
}

\begin{abstract}
We consider the linearized Euler and Maxwell equations and Ohm's law. We calculate the fundamental matrix and give integral representations for the velocity, magnetic induction and pressure. We use the boundary (slip) condition to obtain an integral equation for the jump of the pressure. We give some graphic representations of the velocity and magnetic induction for the case of the flat plate.
\end{abstract}

MSC: Primary 76X05; secondary 45E05; 35E05

Keywords: linearized system; fundamental matrix; integral representation

\section{Introduction}

In papers dedicated to the motion of a wing in an electro-conductive fluid, the lift, drag and moment coefficients were calculated. Recent technological advances claim also for the study of the velocity and electromagnetic fields. We mention two examples: the plasma actuators for aircraft flow control (see [1]) and concealing aircrafts from radar using interaction between ionized gas and electromagnetic radiation. In the present paper, we study the steady two-dimensional flow of an ideal perfectly conducting incompressible fluid around a thin insulating airfoil. We consider the linearized partial differential equations of magnetohydrodynamics (consisting of Euler's and Maxwell's equations and Ohm's law) and calculate the corresponding fundamental matrix. In order to obtain the integral representations for the velocity, the magnetic induction and the pressure fields (which represent the original result of this work), we perform the convolution of the components of the fundamental matrix with the simple layer distributions determined by the jump of the functions we are looking for. We notice that every integral representation has an elliptic as well as a hyperbolic part, this last one being determined by the presence of simple waves bounded by straight characteristics (weak shocks). From the integral representation of the velocity and the boundary conditions (linearized slipping condition and the continuity of the magnetic induction), we rediscover the singular integral equation for the jump of the pressure across the airfoil. We consider the particular case of the flat plate for which the solution of the integral equation is known. Then we perform some numerical integrations to calculate the velocity and the magnetic induction at the points of a two-dimensional grid in order to provide graphic representations for the velocity and magnetic induction fields.

(c)2013 Carabineanu; licensee Springer. This is an Open Access article distributed under the terms of the Creative Commons Attribution License (http://creativecommons.org/licenses/by/2.0), which permits unrestricted use, distribution, and reproduction in any medium, provided the original work is properly cited. 


\section{Fundamental matrix of the linearized equations for the two-dimensional incompressible flow of perfectly conducting fluids}

Let $\mathbf{v}, \mathbf{b}$ and $p$ designate the nondimensional perturbations of the velocity, magnetic induction and pressure, respectively, determined by the presence of a thin insulating airfoil whose equation is

$$
y=h_{ \pm}(x), \quad x \in[0,1], \quad\left|h_{ \pm}(x)\right| \ll 1, \quad\left|h_{ \pm}^{\prime}(x)\right| \ll 1
$$

At infinity, we assume that the unperturbed motion is uniform and parallel to the $O x$-axis and that there exists a homogeneous magnetic field whose nondimensional expression is

$$
\mathbf{B}_{0}=\left(\alpha_{x}, \alpha_{y}\right), \quad \alpha_{x}=\cos (\alpha), \quad \alpha_{y}=\sin (\alpha), \quad \alpha \in(-\pi / 2, \pi / 2) .
$$

As it is shown in [2], Section 5.2, $\mathbf{v}=\left(v_{x}, v_{y}\right), \mathbf{b}=\left(b_{x}, b_{y}\right)$ and $p$ satisfy the following system of linear partial differential equations obtained by means of the small perturbations technique:

$$
\begin{aligned}
& \frac{\partial v_{x}}{\partial x}+\frac{\partial p}{\partial x}+\frac{\alpha_{y}}{A}\left(\frac{\partial b_{y}}{\partial x}-\frac{\partial b_{x}}{\partial y}\right)=0, \\
& \frac{\partial v_{y}}{\partial x}+\frac{\partial p}{\partial y}-\frac{\alpha_{x}}{A}\left(\frac{\partial b_{y}}{\partial x}-\frac{\partial b_{x}}{\partial y}\right)=0 \\
& \frac{\partial v_{x}}{\partial x}+\frac{\partial v_{y}}{\partial y}=0 \\
& \frac{\partial b_{x}}{\partial x}+\frac{\partial b_{y}}{\partial y}=0 \\
& b_{y}+\alpha_{y} v_{x}-\alpha_{x} v_{y}=0
\end{aligned}
$$

with $A=\frac{1}{\sqrt{R h}}$ being Alfvén's number ( $R h$ is the magnetic pressure number). We introduce like in [3] the fundamental matrix of linear system (3)

$$
\left(\begin{array}{lllll}
v_{x}^{(1)} & v_{y}^{(1)} & b_{x}^{(1)} & b_{y}^{(1)} & p^{(1)} \\
v_{x}^{(2)} & v_{y}^{(2)} & b_{x}^{(2)} & b_{y}^{(2)} & p^{(2)} \\
v_{x}^{(3)} & v_{y}^{(3)} & b_{x}^{(3)} & b_{y}^{(3)} & p^{(3)} \\
v_{x}^{(4)} & v_{y}^{(4)} & b_{x}^{(4)} & b_{y}^{(4)} & p^{(4)}
\end{array}\right)
$$

whose components are the fundamental solutions of the systems

$$
\begin{aligned}
& \frac{\partial v_{x}^{(j)}}{\partial x}+\frac{\partial p^{(j)}}{\partial x}+\frac{\alpha_{y}}{A}\left(\frac{\partial b_{y}^{(j)}}{\partial x}-\frac{\partial b_{x}^{(j)}}{\partial y}\right)=\delta_{j 1} \delta(x, y), \\
& \frac{\partial v_{y}^{(j)}}{\partial x}+\frac{\partial p^{(j)}}{\partial y}-\frac{\alpha_{x}}{A}\left(\frac{\partial b_{y}^{(j)}}{\partial x}-\frac{\partial b_{x}^{(j)}}{\partial y}\right)=\delta_{j 2} \delta(x, y), \\
& \frac{\partial v_{x}^{(j)}}{\partial x}+\frac{\partial v_{y}^{(j)}}{\partial y}=\delta_{j 3} \delta(x, y)
\end{aligned}
$$




$$
\begin{aligned}
& \frac{\partial b_{x}^{(j)}}{\partial x}+\frac{\partial b_{y}^{(j)}}{\partial y}=\delta_{j 4} \delta(x, y), \\
& b_{y}^{(j)}+\alpha_{y} v_{x}^{(j)}-\alpha_{x} v_{y}^{(j)}=0
\end{aligned}
$$

where $\delta(x, y)$ is Dirac's distribution and $\delta_{j i}= \begin{cases}1, & j=i, \\ 0, & j \neq i\end{cases}$

\section{Some components of the fundamental matrix}

We shall use the Fourier transform

$$
F[f]\left(\xi_{1}, \xi_{2}\right)=\iint_{\mathbf{R}^{2}} \exp \left[i\left(x \xi_{1}+y \xi_{2}\right)\right] f(x, y) d x d y
$$

Taking into account that

$$
\begin{aligned}
& F\left[\frac{\partial f}{\partial x}\right]\left(\xi_{1}, \xi_{2}\right)=-i \xi_{1} F[f]\left(\xi_{1}, \xi_{2}\right), \quad F\left[\frac{\partial f}{\partial y}\right]\left(\xi_{1}, \xi_{2}\right)=-i \xi_{2} F[f]\left(\xi_{1}, \xi_{2}\right), \\
& F[\delta]=1
\end{aligned}
$$

and using the notation $F[f]=\tilde{f}$, we obtain from (5)

$$
\begin{aligned}
& -i \xi_{1} \tilde{v}_{x}^{(j)}-i \xi_{1} \tilde{p}^{(j)}+\frac{i \alpha_{y} \xi_{2}}{A^{2}} \tilde{b}_{x}^{(j)}-\frac{i \alpha_{y} \xi_{1}}{A^{2}} \tilde{b}_{y}^{(j)}=\delta_{j 1}, \\
& -i \xi_{1} \tilde{v}_{y}^{(j)}-i \xi_{2} \tilde{p}^{(j)}-\frac{i \alpha_{x} \xi_{2}}{A^{2}} \tilde{b}_{x}^{(j)}+\frac{i \alpha_{x} \xi_{1}}{A^{2}} \tilde{b}_{y}^{(j)}=\delta_{j 2}, \\
& -i \xi_{1} \tilde{v}_{x}^{(j)}-i \xi_{2} \tilde{v}_{y}^{(j)}=\delta_{j 3}, \\
& -i \xi_{1} \tilde{b}_{x}^{(j)}-i \xi_{2} \tilde{b}_{y}^{(j)}=\delta_{j 4}, \\
& \tilde{b}_{y}^{(j)}+\alpha_{y} \tilde{v}_{x}^{(j)}-\alpha_{x} \tilde{v}_{y}^{(j)}=0 .
\end{aligned}
$$

Solving system (9), we get for $j=2$

$$
\begin{aligned}
& \tilde{v}_{x}^{(2)}=\frac{i \xi_{1}^{2} \xi_{2}}{\left(\xi_{1}^{2}+\xi_{2}^{2}\right)\left(\left(\frac{\xi_{1} \alpha_{x}+\xi_{2} \alpha_{y}}{A}\right)^{2}-\xi_{1}^{2}\right)}, \\
& \tilde{v}_{y}^{(2)}=\frac{-i \xi_{1}^{3}}{\left(\xi_{1}^{2}+\xi_{2}^{2}\right)\left(\left(\frac{\xi_{1} \alpha_{x}+\xi_{2} \alpha_{y}}{A}\right)^{2}-\xi_{1}^{2}\right)}, \\
& \tilde{b}_{x}^{(2)}=\frac{i \alpha_{x} \xi_{1}^{2} \xi_{2}+i \alpha_{y} \xi_{1} \xi_{2}^{2}}{\left(\xi_{1}^{2}+\xi_{2}^{2}\right)\left(\left(\frac{\xi_{1} \alpha_{x}+\xi_{2} \alpha_{y}}{A}\right)^{2}-\xi_{1}^{2}\right)}, \\
& \tilde{p}^{(2)}=-i \frac{\xi_{1}^{2} \xi_{2}}{\left(\xi_{1}^{2}+\xi_{2}^{2}\right)\left(\left(\frac{\xi_{1} \alpha_{x}+\xi_{2} \alpha_{y}}{A}\right)^{2}-\xi_{1}^{2}\right)}+i \frac{\alpha_{y}}{A^{2}} \frac{\xi_{1} \alpha_{x}+\xi_{2} \alpha_{y}}{\left(\frac{\xi_{1} \alpha_{x}+\xi_{2} \alpha_{y}}{A}\right)^{2}-\xi_{1}^{2}} .
\end{aligned}
$$

For $(l, k) \in\{(3,0) ;(2,1) ;(1,2)\}$, we have

$$
\frac{\xi_{1}^{l} \xi_{2}^{k}}{\left(\xi_{1}^{2}+\xi_{2}^{2}\right)\left(\left(\frac{\xi_{1} \alpha_{x}+\xi_{2} \alpha_{y}}{A}\right)^{2}-\xi_{1}^{2}\right)}=\frac{a_{l k} \xi_{1}+b_{l k} \xi_{2}}{\xi_{1}^{2}+\xi_{2}^{2}}+\frac{c_{l k} \xi_{1}+d_{l k} \xi_{2}}{\left(\frac{\xi_{1} \alpha_{x}+\xi_{2} \alpha_{y}}{A}\right)^{2}-\xi_{1}^{2}}
$$


with

$$
\begin{array}{ll}
a_{30}=-\frac{A^{2}\left(A^{2}+1-2 \alpha_{x}^{2}\right)}{1+2 A^{2}\left(1-2 \alpha_{x}^{2}\right)+A^{4}}, & b_{30}=-\frac{2 A^{2} \alpha_{x} \alpha_{y}}{1+2 A^{2}\left(1-2 \alpha_{x}^{2}\right)+A^{4}}, \\
c_{30}=\frac{\alpha_{y}^{2}\left(A^{2}+1+2 \alpha_{x}^{2}\right)}{1+2 A^{2}\left(1-2 \alpha_{x}^{2}\right)+A^{4}}, & d_{30}=\frac{2 \alpha_{x} \alpha_{y}^{3}}{1+2 A^{2}\left(1-2 \alpha_{x}^{2}\right)+A^{4}}, \\
a_{21}=\frac{2 A^{2} \alpha_{x} \alpha_{y}}{1+2 A^{2}\left(1-2 \alpha_{x}^{2}\right)+A^{4}}, & b_{21}=-\frac{A^{2}\left(A^{2}+1-2 \alpha_{x}^{2}\right)}{1+2 A^{2}\left(1-2 \alpha_{x}^{2}\right)+A^{4}}, \\
c_{21}=\frac{2 \alpha_{x} \alpha_{y}\left(A^{2}-\alpha_{x}^{2}\right)}{1+2 A^{2}\left(1-2 \alpha_{x}^{2}\right)+A^{4}}, & d_{21}=\frac{\alpha_{y}^{2}\left(A^{2}+1-2 \alpha_{x}^{2}\right)}{1+2 A^{2}\left(1-2 \alpha_{x}^{2}\right)+A^{4}}, \\
a_{12}=\frac{A^{2}\left(A^{2}+1-2 \alpha_{x}^{2}\right)}{1+2 A^{2}\left(1-2 \alpha_{x}^{2}\right)+A^{4}}, & b_{12}=\frac{2 A^{2} \alpha_{x} \alpha_{y}}{1+2 A^{2}\left(1-2 \alpha_{x}^{2}\right)+A^{4}}, \\
c_{12}=\frac{\left(A^{2}-\alpha_{x}^{2}\right)\left(A^{2}+1-2 \alpha_{x}^{2}\right)}{1+2 A^{2}\left(1-2 \alpha_{x}^{2}\right)+A^{4}}, & d_{12}=-\frac{2 \alpha_{x} \alpha_{y}^{3}}{1+2 A^{2}\left(1-2 \alpha_{x}^{2}\right)+A^{4}} .
\end{array}
$$

Hence

$$
\begin{aligned}
& \tilde{v}_{x}^{(2)}=\frac{i a_{21} \xi_{1}+i b_{21} \xi_{2}}{\xi_{1}^{2}+\xi_{2}^{2}}+\frac{i c_{21} \xi_{1}+i d_{21} \xi_{2}}{\left(\frac{\xi_{1} \alpha_{x}+\xi_{2} \alpha_{y}}{A}\right)^{2}-\xi_{1}^{2}}, \\
& \tilde{v}_{y}^{(2)}=\frac{-i a_{30} \xi_{1}-i b_{30} \xi_{2}}{\xi_{1}^{2}+\xi_{2}^{2}}+\frac{-i c_{30} \xi_{1}-i d_{30} \xi_{2}}{\left(\frac{\xi_{1} \alpha_{x}+\xi_{2} \alpha_{y}}{A}\right)^{2}-\xi_{1}^{2}}, \\
& \tilde{b}_{x}^{(2)}=\alpha_{x} \tilde{v}_{x}^{(2)}+\alpha_{y} \tilde{v}_{y}^{(2)}+\frac{i \alpha_{y} \xi_{1}}{\left(\frac{\xi_{1} \alpha_{x}+\xi_{2} \alpha_{y}}{A}\right)^{2}-\xi_{1}^{2}}, \\
& \tilde{p}^{(2)}=-\tilde{v}_{x}^{(2)}+\frac{i \alpha_{y}}{A^{2}} \frac{\xi_{1} \alpha_{x}+\xi_{2} \alpha_{y}}{\left(\frac{\xi_{1} \alpha_{x}+\xi_{2} \alpha_{y}}{A}\right)^{2}-\xi_{1}^{2}} .
\end{aligned}
$$

Using the inverse Fourier transforms ([4], Appendix 1)

$$
\begin{aligned}
& F^{-1}\left[\frac{\xi_{1}}{\xi_{1}^{2}+\xi_{2}^{2}}\right]=\frac{-i}{2 \pi} \frac{\partial}{\partial x} \ln \sqrt{x^{2}+y^{2}}, \\
& F^{-1}\left[\frac{\xi_{2}}{\xi_{1}^{2}+\xi_{2}^{2}}\right]=\frac{-i}{2 \pi} \frac{\partial}{\partial y} \ln \sqrt{x^{2}+y^{2}}, \\
& F^{-1}\left[\frac{\xi_{1}}{\left(\frac{\xi_{1} \alpha_{x}+\xi_{2} \alpha_{y}}{A}\right)^{2}-\xi_{1}^{2}}\right]=\frac{i A}{2 \alpha_{y}} \frac{\partial}{\partial x} H\left(x-\frac{\alpha_{x}}{\alpha_{y}} y-\frac{A}{\alpha_{y}}|y|\right), \\
& F^{-1}\left[\frac{\xi_{2}}{\left(\frac{\xi_{1} \alpha_{x}+\xi_{2} \alpha_{y}}{A}\right)^{2}-\xi_{1}^{2}}\right]=\frac{i A}{2 \alpha_{y}} \frac{\partial}{\partial y} H\left(x-\frac{\alpha_{x}}{\alpha_{y}} y-\frac{A}{\alpha_{y}}|y|\right)
\end{aligned}
$$

(where $H(x)=\left\{\begin{array}{ll}1, & x \geq 0, \\ 0, & x<0\end{array}\right.$ is Heaviside's function), we get

$$
\begin{aligned}
v_{x}^{(2)}= & \frac{a_{21}}{2 \pi} \frac{x}{x^{2}+y^{2}}+\frac{b_{21}}{2 \pi} \frac{y}{x^{2}+y^{2}} \\
& -\frac{A c_{21}}{2 \alpha_{y}} \frac{\partial}{\partial x} H\left(x-\frac{\alpha_{x}}{\alpha_{y}} y-\frac{A}{\alpha_{y}}|y|\right)-\frac{A d_{21}}{2 \alpha_{y}} \frac{\partial}{\partial y} H\left(x-\frac{\alpha_{x}}{\alpha_{y}} y-\frac{A}{\alpha_{y}}|y|\right),
\end{aligned}
$$




$$
\begin{aligned}
v_{y}^{(2)}= & -\frac{a_{30}}{2 \pi} \frac{x}{x^{2}+y^{2}}-\frac{b_{30}}{2 \pi} \frac{y}{x^{2}+y^{2}} \\
& +\frac{A c_{30}}{2 \alpha_{y}} \frac{\partial}{\partial x} H\left(x-\frac{\alpha_{x}}{\alpha_{y}} y-\frac{A}{\alpha_{y}}|y|\right)+\frac{A d_{30}}{2 \alpha_{y}} \frac{\partial}{\partial y} H\left(x-\frac{\alpha_{x}}{\alpha_{y}} y-\frac{A}{\alpha_{y}}|y|\right), \\
b_{x}^{(2)}= & \alpha_{x} v_{x}^{(2)}+\alpha_{y} v_{y}^{(2)}-\frac{A}{2} \frac{\partial}{\partial x} H\left(x-\frac{\alpha_{x}}{\alpha_{y}} y-\frac{A}{\alpha_{y}}|y|\right), \\
p_{x}^{(2)=} & -v_{x}^{(2)}-\frac{\alpha_{x}}{2 A} \frac{\partial}{\partial x} H\left(x-\frac{\alpha_{x}}{\alpha_{y}} y-\frac{A}{\alpha_{y}}|y|\right)-\frac{\alpha_{y}}{2 A} \frac{\partial}{\partial y} H\left(x-\frac{\alpha_{x}}{\alpha_{y}} y-\frac{A}{\alpha_{y}}|y|\right) .
\end{aligned}
$$

\section{Integral representations}

In thin airfoil theory, the linearized boundary conditions are usually imposed on the segment $[-1,1]$ and the functions we are looking for are defined on $\mathbf{R}^{2} \backslash[-1,1]$. Since $v_{x}, v_{y}, b_{x}$, $b_{y}$ and $p$ are integrable functions, they may be regarded as regular distributions. Taking into account the boundary conditions ([2], Chapter 5)

$$
[\mathbf{b}](x)=0, \quad v_{y}(x, \pm 0)=h_{ \pm}^{\prime}(x), \quad x \in[-1,1]
$$

we obtain the following linearized system for the distributions $v_{x}, v_{y}, b_{x}, b_{y}$ and $p$ :

$$
\begin{aligned}
& \frac{\partial v_{x}}{\partial x}+\frac{\partial p}{\partial x}+\frac{\alpha_{y}}{A}\left(\frac{\partial b_{y}}{\partial x}-\frac{\partial b_{x}}{\partial y}\right)=0=f_{1}, \\
& \frac{\partial v_{y}}{\partial x}+\frac{\partial p}{\partial y}-\frac{\alpha_{x}}{A}\left(\frac{\partial b_{y}}{\partial x}-\frac{\partial b_{x}}{\partial y}\right)=[p] \delta_{[-1,1]}=f_{2}, \\
& \frac{\partial v_{x}}{\partial x}+\frac{\partial v_{y}}{\partial y}=\left[h^{\prime}\right] \delta_{[-1,1]}=f_{3}, \\
& \frac{\partial b_{x}}{\partial x}+\frac{\partial b_{y}}{\partial y}=0=f_{4}, \\
& b_{y}+\alpha_{y} v_{x}-\alpha_{x} v_{y}=0,
\end{aligned}
$$

where $[\mathbf{b}](x)$ and $[p](x)$ represent the jumps of $\mathbf{b}$ and $p$ over the segment $[-1,1]$ and $[p] \delta_{[-1,1]},\left[h^{\prime}\right] \delta_{[-1,1]}$ are simple layer distributions. We may easily verify that

$$
\begin{array}{ll}
v_{x}=\sum_{j=1}^{4} v_{x}^{(j)} \star f_{j}, & v_{y}=\sum_{j=1}^{4} v_{y}^{(j)} \star f_{j}, \\
b_{x}=\sum_{j=1}^{4} b_{x}^{(j)} \star f_{j}, & p=\sum_{j=1}^{4} p^{(j)} \star f_{j},
\end{array}
$$

where $\star$ stands for the convolution product. We shall consider, for the sake of simplicity, the case of zero thickness wing, i.e., $h_{+}(x)=h_{-}(x)$. Hence $f_{1}=f_{3}=f_{4}=0$ and

$$
\begin{array}{ll}
v_{x}=v_{x}^{(2)} \star[p] \delta_{[-1,1]}, & v_{y}=v_{y}^{(2)} \star[p] \delta_{[-1,1]}, \\
b_{x}=b_{x}^{(2)} \star[p] \delta_{[-1,1]}, & p=p^{(2)} \star[p] \delta_{[-1,1]} .
\end{array}
$$


We calculate the convolutions and obtain the following representation for $v_{x}$ :

$$
\begin{aligned}
\nu_{x}(x, y)= & \frac{a_{21}}{2 \pi} \int_{-1}^{1}[p](\xi) \frac{x-\xi}{(x-\xi)^{2}+y^{2}} d \xi+\frac{b_{21}}{2 \pi} \int_{-1}^{1}[p](\xi) \frac{y}{(x-\xi)^{2}+y^{2}} d \xi \\
& -\frac{A c_{21}}{2 \alpha_{y}} \frac{\partial}{\partial x} \int_{-1}^{1}[p](\xi) H\left(x-\xi-\frac{\alpha_{x}}{\alpha_{y}} y-\frac{A}{\alpha_{y}}|y|\right) d \xi \\
& -\frac{A d_{21}}{2 \alpha_{y}} \frac{\partial}{\partial y} \int_{-1}^{1}[p(\xi)] H\left(x-\xi-\frac{\alpha_{x}}{\alpha_{y}} y-\frac{A}{\alpha_{y}}|y|\right) d \xi \\
= & \frac{a_{21}}{2 \pi} \int_{-1}^{1}[p](\xi) \frac{x-\xi}{(x-\xi)^{2}+y^{2}} d \xi+\frac{b_{21}}{2 \pi} \int_{-1}^{1}[p](\xi) \frac{y}{(x-\xi)^{2}+y^{2}} d \xi \\
& -\frac{A}{2 \alpha_{y}^{2}}\left(c_{21} \alpha_{y}-d_{21} \alpha_{x}-d_{21} A \frac{y}{|y|}\right) \\
& \times[p]\left(x-\frac{\alpha_{x}}{\alpha_{y}} y-\frac{A}{\alpha_{y}}|y|\right) \times H\left(1-\left|x-\frac{\alpha_{x}}{\alpha_{y}} y-\frac{A}{\alpha_{y}}\right| y||\right) .
\end{aligned}
$$

Similarly, we get

$$
\begin{aligned}
v_{y}(x, y)= & -\frac{a_{30}}{2 \pi} \int_{-1}^{1}[p](\xi) \frac{x-\xi}{(x-\xi)^{2}+y^{2}} d \xi-\frac{b_{30}}{2 \pi} \int_{-1}^{1}[p](\xi) \frac{y}{(x-\xi)^{2}+y^{2}} d \xi \\
& +\frac{A}{2 \alpha_{y}^{2}}\left(c_{30} \alpha_{y}-d_{30} \alpha_{x}-d_{30} A \frac{y}{|y|}\right) \\
& \times[p]\left(x-\frac{\alpha_{x}}{\alpha_{y}} y-\frac{A}{\alpha_{y}}|y|\right) \times H\left(1-\left|x-\frac{\alpha_{x}}{\alpha_{y}} y-\frac{A}{\alpha_{y}}\right| y||\right), \\
b_{x}(x, y)= & \alpha_{x} v_{x}(x, y)+\alpha_{y} v_{y}(x, y) \\
& -\frac{A}{2}[p]\left(x-\frac{\alpha_{x}}{\alpha_{y}} y-\frac{A}{\alpha_{y}}|y|\right) \times H\left(1-\left|x-\frac{\alpha_{x}}{\alpha_{y}} y-\frac{A}{\alpha_{y}}\right| y||\right), \\
p(x, y)= & -v_{x}(x, y) \\
& +\frac{y}{2|y|}[p]\left(x-\frac{\alpha_{x}}{\alpha_{y}} y-\frac{A}{\alpha_{y}}|y|\right) \times H\left(1-\left|x-\frac{\alpha_{x}}{\alpha_{y}} y-\frac{A}{\alpha_{y}}\right| y||\right) .
\end{aligned}
$$

\section{The integral equation for the jump of the pressure}

Using the Plemelj formulas and linearized boundary conditions (19), we get from (23) the integral equation

$$
\begin{aligned}
h^{\prime}(x) & =v_{y}(x, \pm 0) \\
& =-\frac{a_{30}}{2 \pi} p \cdot v \cdot \int_{-1}^{1} \frac{[p(\xi)]}{x-\xi} d \xi+\frac{A\left(c_{30} \alpha_{y}-d_{30} \alpha_{x}\right)}{2 \alpha_{y}^{2}}[p(x)], \quad x \in(-1,1),
\end{aligned}
$$

which is equivalent to

$$
-\beta[p](x)+\frac{k}{\pi} p \cdot v \cdot \int_{-1}^{1} \frac{[p](\xi)}{\xi-x} d \xi=\chi h^{\prime}(x),
$$


where $k=A^{2}+1-2 \alpha_{x}^{2}, \beta=\alpha_{y}\left(1+A^{2}\right) A^{-1}$ and $\chi=2\left(4 \alpha_{x}^{2}+A^{-2}-A^{2}\right)$. As it is shown in [3, 5] and [2], Chapter 5 , the solution of equation (27) is

$$
\begin{aligned}
{[p](x)=} & \frac{-\beta \chi}{\beta^{2}+k^{2}} h^{\prime}(x)-\frac{k \chi}{\pi\left(\beta^{2}+k^{2}\right)}\left(\frac{1-x}{1+x}\right)^{\theta} p \cdot v \cdot \int_{-1}^{1}\left(\frac{1+\xi}{1-\xi}\right)^{\theta} \frac{h^{\prime}(\xi)}{\xi-x} d \xi \\
& +\frac{2 C \sin \theta \pi}{(1-x)^{1-\theta}(1+x)^{\theta}}, \quad \tan \theta \pi=\frac{k}{\beta}, 0 \leq \theta<1 .
\end{aligned}
$$

We may take $C=0$ if we impose Kutta-Joukovsky's condition. Other choices of the constant $C$ were considered by Stewartson [6].

\section{The flat plate}

In this case, $h(x)=-\varepsilon x, h^{\prime}(x)=-\varepsilon$. Taking into account that

$$
\text { p.v. } \int_{-1}^{1}\left(\frac{1+\xi}{1-\xi}\right)^{\theta} \frac{1}{\xi-x} d \xi=\frac{\pi}{\sin \theta \pi}\left\{1-\left(\frac{1+x}{1-x}\right)^{\theta} \cos \theta \pi\right\}
$$

we get from (28)

$$
[p](x)=\frac{\varepsilon \chi}{\sqrt{\beta^{2}+k^{2}}}\left(\frac{1-x}{1+x}\right)^{\theta} .
$$

The lift is ([2], 5.2.6)

$$
L=-\int_{-1}^{1}[p](x) d x=4 \varepsilon \pi \frac{\left(A^{2}-1\right)^{2}+4 A^{2} \alpha_{y}^{2}}{A^{2}\left(A^{2}-1+2 \alpha_{y}^{2}\right)} \theta .
$$

In order to obtain graphic representations for the velocity (Figure 1), magnetic induction (Figure 2) and lift (Figure 3), we use the quadrature formulas

$$
\begin{aligned}
& I_{1}(x, y)=\int_{-1}^{1}\left(\frac{1-\xi}{1+\xi}\right)^{\theta} \frac{x-\xi}{(x-\xi)^{2}+y^{2}}=\sum_{k=1}^{N} W_{k} \frac{x-t_{k}}{\left(x-t_{k}\right)^{2}+y^{2}} \\
& I_{2}=\int_{-1}^{1}\left(\frac{1-\xi}{1+\xi}\right)^{\theta} \frac{y}{(x-\xi)^{2}+y^{2}}=\sum_{k=1}^{N} W_{k} \frac{y}{\left(x-t_{k}\right)^{2}+y^{2}}
\end{aligned}
$$

where

$$
W_{k}=-\frac{4 \Gamma(N+\theta+1) \Gamma(N-\theta+1)}{[(N+1) !]^{2} P_{N-1}^{(\theta+1,-\theta+1)}\left(t_{k}\right) P_{N+1}^{(\theta,-\theta)}\left(t_{k}\right)} .
$$

$P_{N-1}^{(\theta+1,-\theta+1)}\left(t_{k}\right), P_{N+1}^{(\theta,-\theta)}\left(t_{k}\right)$ are the Jacobi polynomials and $t_{k}, k=1, \ldots, N$, are the roots of $P_{N}^{(\theta,-\theta)}\left(t_{k}\right)$. We considered $\varepsilon=0.2$.

\section{Conclusions}

We have calculated the velocity, the pressure and the magnetic induction for the steady flow of an incompressible perfectly conducting fluid past a thin airfoil. The integral rep- 

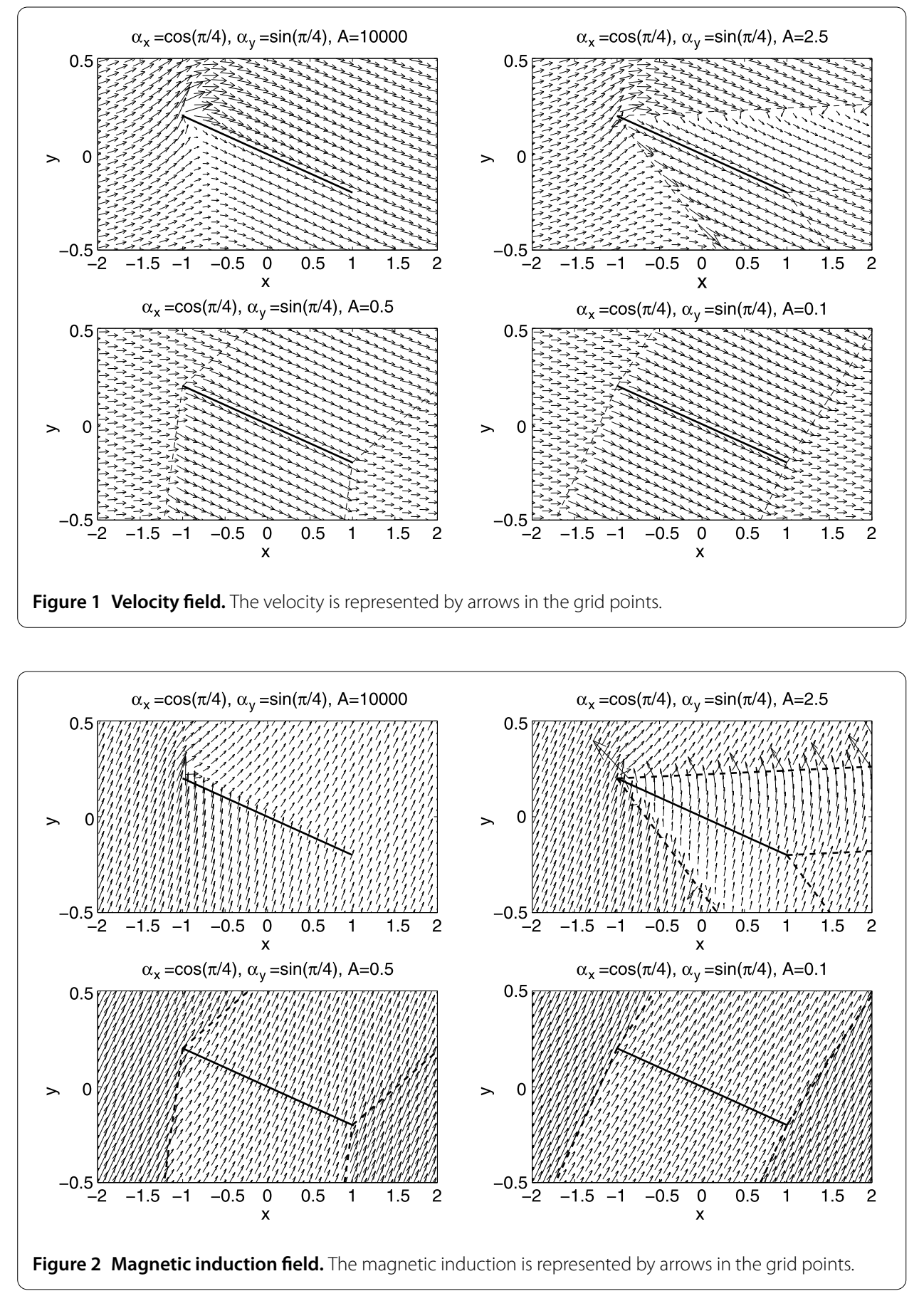

resentations of the velocity, pressure and magnetic induction contain respectively an elliptic part and a hyperbolic one. Some calculations were performed for the flat plate. In the graphic representation of the velocity and magnetic induction from Figures 1 and 2, we may observe the simple waves which are determined by the influence of the magnetic field. In Figure 3 we represent the lift coefficient against Alfvén's number $A$. We notice that the lift coefficient increases when $A$ decreases, i.e., when the value of the magnetic induction at infinity increases. 


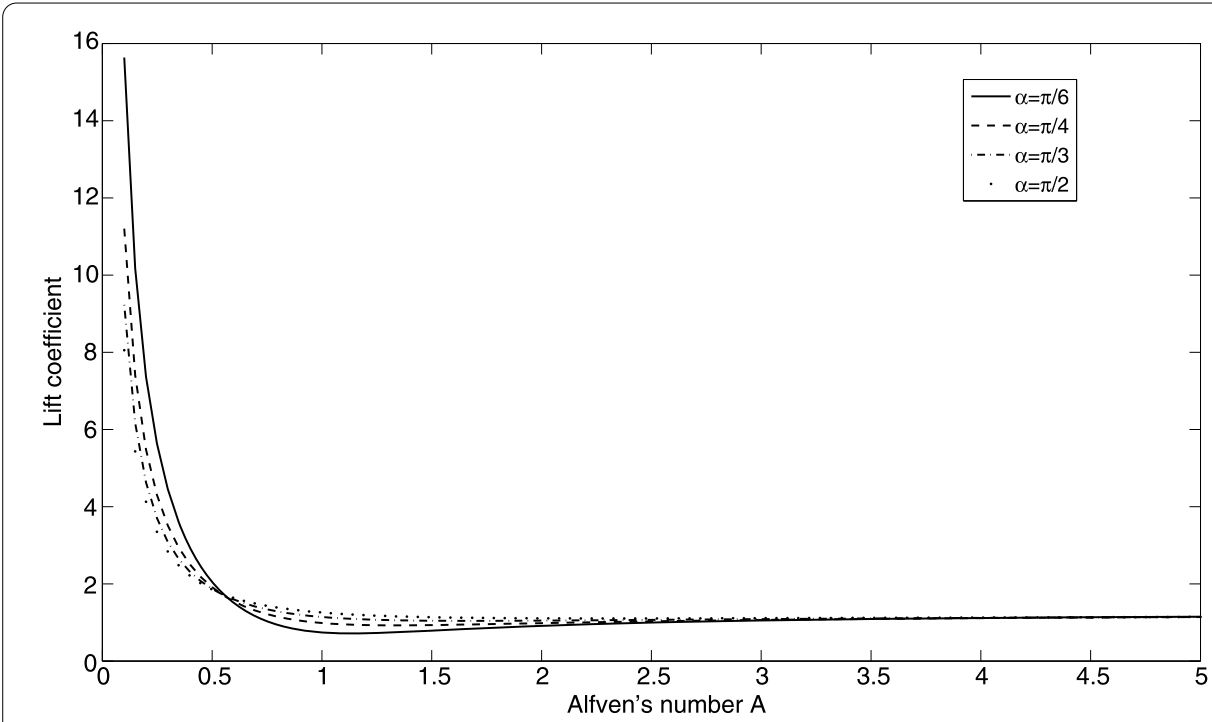

Figure 3 Lift coefficient versus Alfvén's number. The lift coefficient is calculated for various values of the angle $\alpha$.

\section{Competing interests}

The author declares that he has no competing interests.

Received: 23 August 2013 Accepted: 21 October 2013 Published: 08 Nov 2013

\section{References}

1. Cork, TC, Post, ML, Orlov, DM: Single dielectric barrier discharge plasma enhanced aerodynamics: physics, modeling and applications. Exp. Fluids 46, 1-26 (2009)

2. Dragoş, L: Magnetofluid Dynamics. Ed. Academiei Române, Bucharest (1975)

3. Carabineanu, A: Fundamental solutions for the plane-parallel steady flow of incompressible perfectly conducting fluids past thin airfoils. Rev. Roum. Math. Pures Appl. 40, 279-288 (1995)

4. Dragoş, L: Mathematical Methods in Aerodynamics. Kluwer Academic, Dordrecht (2003)

5. Homentcovschi, D: Sur la résolution explicite du problème de Hilbert. Application au calcul de la portance d'un profil mince dans un fluide électroconducteur. Rev. Roum. Math. Pures Appl. 14, 203-223 (1969)

6. Stewartson, K: Magneto-fluid dynamics of thin bodies in oblique fields. Z. Angew. Math. Phys. 12, 261-271 (1961)

10.1186/1687-2770-2013-231

Cite this article as: Carabineanu: Steady flow of an incompressible perfectly conducting fluid past a thin airfoil.

Boundary Value Problems 2013, 2013:231

\section{Submit your manuscript to a SpringerOpen ${ }^{\circ}$ journal and benefit from:}

- Convenient online submission

Rigorous peer review

- Immediate publication on acceptance

- Open access: articles freely available online

- High visibility within the field

- Retaining the copyright to your article

Submit your next manuscript at $>$ springeropen.com 